\section{LA FILOSOFÍA DE LA CIENCIA Y EL LENGUAJE: RELACIONES CAMBIANTES, ALCANCES Y LÍMITES}

\author{
Pablo Lorenzano \\ Universidad Nacional de Quilmes \\ Consejo Nacional de Investigaciones Científicas y Técnicas \\ Argentina
}

\begin{abstract}
This paper consists of three sections. In the first one, some of the main developments in the philosophy of science through the $X X$ century up to the present will be pointed out, and inserted them in the frame of some more general philosophical transformations, such as the so-called "linguistic turn" and "pragmatic turn", respectively. In the second one, the established connection will be nuanced, from a revision of the work of a "classical" author such as Carnap. Finally, it will be intended a kind of "balance and future perspectives".
\end{abstract}

KEY WORDS: Language; science; linguistic turn; pragmatic turn; Carnap; structuralist view of theories.

\section{El "giro lingüístico", el "giro pragmático" Y LA FILOSOFÍA DE LA CIENCIA}

Podria decirse que la filosofía de la ciencia surge como disciplina con especificidad propia, profesionalizándose, en el período de entreguerras. Esta profesionalización se da a partir de la conformación de lo que desde Carnap, Neurath \& Hahn (1929) pasaría a denominarse oficialmente Círculo de Viena', y se consolida tras la llegada a los Estados Unidos de los principales filósofos de la ciencia centroeuropeos. En su desarrollo desde entonces, podemos señalar tres períodos, etapas o fases principales por las que ha atravesado la filosofía de la ciencia: (1) una fase clásica, que abarca desde fines de los años veinte hasta finales de los años sesenta, en la que se establece la llamada concepción heredada; (2) una fase historicista, iniciada en los años sesenta y dominante durante los años setenta y principios de los ochenta; y (3) una fase contemporánea, que se inicia a comienzos de los años setenta y se extiende hasta nuestros días.

\section{PHILOSOPHY OF SCIENCE AND LANGUAGE: CHANGING RELATIONSHIPS, SCOPES AND LIMITS}

RESUMEN: Este trabajo consta de tres secciones. En la primera, se señalarán algunos de los principales desarrollos de la filosofía de la ciencia durante el siglo XX y lo que va del XXI, ubicándolos en el marco de ciertas transformaciones filosóficas más generales, conocidas con los nombres de "giro lingüístico" y "giro pragmático", respectivamente. En la segunda, se matizará la conexión establecida, fundamentalmente a partir de la revisión de la obra de un autor "clásico" como Carnap. Por último, se intentará realizar una suerte de "balance y perspectivas futuras".

PALABRAS CLAVE: Lenguaje; ciencia; giro lingüístico; giro pragmático; Carnap; concepción estructuralista de las teorias.

La filosofía de la ciencia producto del Círculo de Viena y, de este modo, en gran medida, la filosofía de la ciencia de toda la fase clásica, se encontraba influenciada por el análisis lógico del lenguaje. Lo que caracteriza a éste, así como también en general a la filosofía analítica, podría sintetizarse de la siguiente manera:

Lo que distingue a la filosofía analítica en sus diversas manifestaciones de otras escuelas es la creencia, primero, de que un tratamiento filosófico del pensamiento puede ser obtenido a través de un tratamiento filosófico del lenguaje, $y$, segundo, de que un tratamiento comprehensivo puede ser obtenido solamente así. [...] la filosofía analítica nació cuando se tomó el "giro lingüístico" [que se distingue por] la mayor insistencia en el reflejo de los pensamientos por los enunciados. [...] El lenguaje pudiera ser un espejo distorsionador: pero es el único espejo que tenemos (Dummett, 1993, 4-6).

Vemos así que la filosofía clásica de la ciencia se encuentra asociada desde sus comienzos también con aquello que 
habria de denominarse "giro lingüístico". Esta expresión, que luego extendería su aplicación a otros ámbitos de la cultura por fuera de la filosofia, es introducida en 1964 por el filósofo originariamente miembro del Círculo de Viena Gustav Bergmann, en los siguientes términos:

Todos los filósofos lingüisticos hablan acerca del mundo por medio de un hablar sobre un lenguaje apropiado. Este es el giro lingüistico, la táctica fundamental a manera de método, sobre el que están de acuerdo los filósofos del lenguaje ordinario [del tipo del "segundo" Wittgenstein, Austin y Strawson] y los filósofos del lenguaje ideal [del tipo del "primer" Wittgenstein, Russell y Carnap] (FLO, FLI) (Bergmann, 1964, 177).

Sin embargo, la influencia que se deja sentir por parte del análisis lógico del lenguaje y el giro lingüistico en la filosofía clásica de la ciencia no es a través del mero énfasis en la centralidad en general del lenguaje (científico, en este caso), sino en el modo específico en que lo hace. Para verlo, recordemos que, dentro de la semiótica, la teoría general de los signos y los lenguajes, se suelen distinguir, a partir de la identificación de tres (o cuatro) factores principales en una aplicación de lenguaje (el hablante o usuario del lenguaje, la expresión emitida o las expresiones lingüisticas por él utilizadas y el designatum o denotatum de la expresión o expresiones, sean los significados de las palabras que ocurren y el sentido de los enunciados construibles en el lenguaje o las cosas, clases, relaciones, etc., a las que se refiere el hablante con las expresiones por él proferidas), tres campos: el de la pragmática, el de la semántica y el de la sintaxis. Una investigación de un lenguaje pertenece a la pragmática si hace explícita referencia al hablante; pertenece a la semántica si refiere a los designata o denotata y no a los hablantes (intensional, si refiere a los significados de las palabras y a los sentidos de los enunciados, o extensional, si refiere a las cosas, clases o relaciones); y pertenece a la sintaxis si no trata ni con los hablantes ni con los designata o denotata sino sólo con las expresiones lingüisticas.

En términos generales, podríamos decir que la filosofía clásica de la ciencia se caracterizó por haber concentrado sus análisis en los campos de la sintaxis y de la semántica de la ciencia. De hecho, Wolfgang Stegmüller (1983a, 1983b), en clara alusión a los "Dos dogmas del empirismo" de Quine (1951), denomina "el tercer dogma del empirismo" a la convicción de acuerdo con la cual para elucidar todos los aspectos fundamentales epistemológicamente relevantes de la ciencia bastan los medios (o instrumentos) de la lógica, o sea, los medios sintácticos y semánticos, y sostiene que dicho dogma es asumido por la filosofía de la ciencia durante la fase clásica.

Además, Stegmüller caracteriza a la filosofía historicista de la ciencia por su adopción del denominado "giro pragmático", que hacia los años sesenta se hacía sentir en la filosofía analítica en general y aun en la filosofía clásica de la ciencia, si bien en ésta bajo la forma de "anomalia", al tener que recurrir a elementos pragmáticos en el análisis formal de la explicación científica (para el caso de las explicaciones inductivo-estadísticas, cuyo tratamiento de su ambigüedad lleva a la introducción del concepto de situación de conocimiento relativizado a una persona y a un tiempo histórico).

Hempel, por su parte, estaría de acuerdo con sendas caracterizaciones y la consiguiente contraposición. De allí que se refiera a la filosofia de la ciencia de la fase historicista mediante las denominaciones de "escuela histórico-sociológica" (Hempel, 1979a, 48), "escuela histórico-socio-lógica" (Hempel, 1979b, 291), "escuela histórico-pragmática" (Hempel, 1979a, 58), "escuela pragmatista" (Hempel, 1979b, 291) y "'comprensión histórico-pragmatista' de la investigación científica, de la cual Thomas Kuhn es el principal proponente" (Hempel, 1981, 402). Frente a la "escuela analítico-empirista" (Hempel, 1979b, 291), al "antinaturalismo de Carnap, Popper y pensadores afines pertenecientes, o cercanos, al Círculo de Viena, quienes sostenían que la tarea propia de la metodología y de la filosofía de la ciencia era proveer 'elucidaciones' o 'reconstrucciones racionales' de la forma y función del razonamiento científico", elucidaciones que proveerían "normas o estándares de racionalidad para la prosecución de la investigación científica" y que fueran "formulados con precisión rigurosa por medio del aparato conceptual de la lógica", encontramos, en el caso de Kuhn, el intento de "proveer un tratamiento descriptivo o 'naturalista' de la práctica de investigación científica real, en sus diversos aspectos psicológicos, históricos y socioculturales" y de "examinar los modos de pensamiento que informaron y dirigieron la investigación, la formación teórica y el cambio teórico en la práctica de investigación científica, pasada y presente" (Hempel, 1993, 7), que también proporcione "un tratamiento normativo o 
prescriptivo al exhibir ciertas características en virtud de la cual [el] desarrollo [real de la ciencia] es calificado como racional" (Hempel, 1979b, 292).

En esa misma línea, podriamos añadir que este tratamiento incluye además, por un lado, la referencia a los grupos sociales que desarrollan dicha práctica, las comunidades científicas, así como también, por el otro, el análisis de ciertos aspectos de la semántica de la ciencia, en particular el análisis del cambio de significado de los términos científicos y la inconmensurabilidad resultante vinculados a aquellos episodios en la historia de la ciencia que suelen denominarse "revoluciones científicas".

En cuanto a la filosofía contemporánea de la ciencia, algunas de sus manifestaciones, deudoras de la filosofía de la ciencia de las fases anteriores, sin descuidar completamente los aspectos sintácticos de la ciencia, pero sin centrar sus esfuerzos en ellos, se han concentrado o bien en los aspectos semánticos o bien en los pragmáticos (aunque no necesariamente en los mismos que en la fase historicista o del mismo modo, $y$, en todo caso, con un mayor nivel de claridad y precisión) o bien en ambos, como en el caso de la concepción estructuralista de las teorias, que, como toda concepción semántica o modeloteórica, hace uso esencial de la noción semántica de modelo, al mismo tiempo que no deja de reconocer la presencia de elementos irreductiblemente pragmáticos e históricamente relativos, ni de intentar desarrollar, junto a los estudios sincrónicos, un enfoque diacrónico sistemático, razones por las cuales más que de "enfoque semántico o modeloteórico sin más", habría que calificarla de "enfoque semántico-pragmático" (Moulines, 2002, 114).

Pero si bien las conexiones establecidas entre las distintas fases por las que ha atravesado la filosofía de la ciencia de los siglos XX y XXI, los distintos campos de la semiótica y los "giros lingüístico" y "pragmático" nos ayudan a obtener una visión de conjunto diferenciada y a captar en términos generales sus similitudes y divergencias, podriamos intentar introducir una imagen más matizada, que nos permita entrever una vinculación más estrecha entre las distintas fases $y$, así, en particular, una mayor presencia de los "clásicos" en la filosofía contemporánea de la ciencia, aun cuando también los límites de sus desarrollos. Esto lo haremos, quizás para sorpresa de algunos, a partir del análisis del papel desempeñado por la pragmática en la obra de Rudolf Carnap, el más influyente representante de la filosofía clásica de la ciencia, con excepción quizás de Karl Popper.

\section{La pragmática en la filosofía cLÁsica de la CIENCIA: el caso de Carnap}

Si quisiéramos mencionar autores de la fase clásica, y aun del Círculo de Viena, que hayan reconocido la presencia de elementos irreductiblemente pragmáticos en la ciencia y que hayan tratado de incorporarlos en sus análisis, se nos vendrian a la mente autores tales como Otto Neurath ("quien sostuvo una concepción completamente pragmática de la filosofía de la ciencia", Hempel, 1991, citado en Wolters, 2003, 117) o Edgar Zilsel (menos conocido que el primero, pero en quien es evidente su interés por la historia de la ciencia o incluso por la historia cum elementos sociales de la ciencia), cuyas obras (Neurath, 1979, 1981, 1991, 1994, 1998; Zilsel, 1918, 1926, 1976, 1992, 2000) son consideradas más "sociológicas" e "históricas" comparadas con la más "formalista" de Rudolf Carnap. Más aún, considerando la obra de Kuhn como representativa del "giro pragmático", Neurath y Zilsel parecerian los miembros del Círculo de Viena más cercanos a su pensamiento ${ }^{2}$.

Asimismo, también encontraríamos el mencionado reconocimiento de elementos pragmáticos en la ciencia y de la incorporación de éstos y consiguiente enriquecimiento del análisis realizado en los desarrollos posteriores de otros miembros connotados de la fase clásica, en especial en Carl Gustav Hempel, otrora miembro del "grupo de Berlín", contraparte del Círculo de Viena, quien emprende el giro pragmático al reconocer la presencia de elementos pragmáticos en la explicación, como fue señalado más arriba, y lo completa, bajo la influencia de Kuhn, al hacerlo también en la racionalidad científica, acompañada del "naturalismo" ya aludido (Hempel, 1979a, 1979b, 1981, 1982, 1983, 1993), lo que le terminará valiendo, y no sin razón, la caracterización de "empirista pragmático" (Wolters, 2003).

Sin embargo, es muy probablemente menos o poco conocido o enfatizado lo que ocurre con Rudolf Carnap. Frente a la visión tradicional de acuerdo con la cual el empirismo 
lógico es empirismo (en particular el empirismo británico clásico) cum lógica (ver Kambartel, 1968, 1, entre muchos otros), los comienzos de Carnap no hay que buscarlos en el empirismo de Hume o de Locke, sino en las escuelas tradicionales de filosofía alemana. Así, por ejemplo, cuando en el borrador a una carta para Dingler del 20 de septiembre de 1920, Carnap señala los autores que se encontraba estudiando, no menciona a ningún empirista clásico, sino a Kant, Riemann, Helmholtz, Mach, Avenarius, Poincaré, Natorp, Ostwald, Einstein y Weyl (citada en Coffa, 1991, 207). En esa misma línea de pensamiento, de acuerdo con Mormann $(2000,41)$, las corrientes filosóficas más importantes que impregnan el pensamiento temprano de Carnap son las del neokantismo de Bauch, Cassirer y Natorp, del positivismo crítico alemán de fines del s. XIX de Helmholtz, Mach y Avenarius, del convencionalismo de Dingler y Poincaré (y en menor medida Duhem), de la fenomenología de Husserl.

Por otro lado, de acuerdo con lo que nos relata en Carnap (1963a), en el invierno de 1921 adopta el giro lingüístico, cuando lee Russell (1914), en donde es descrito el método lógico-analítico de hacer filosofía, que tan vívida impresión le causara, pues formulaba clara y explícitamente una concepción de la filosofía que había mantenido implícitamente desde algún tiempo atrás y que se iba a constituir en guía de su actividad:

El estudio de la lógica llega a ser el estudio central en filosofia: proporciona el método de investigación en filosofia, de la misma manera que las matemáticas se lo proporcionan a la física [...]. Todo este supuesto conocimiento de los sistemas tradicionales debe desaparecer, y un nuevo comienzo debe ser hecho [...] para la grande y creciente comunidad de hombres comprometidos en la búsqueda de la ciencia [...], el nuevo método, ya exitoso en venerables problemas como el número, el infinito, la continuidad, el espacio y el tiempo, debería ejercer una atracción que los antiguos métodos han fallado completamente en ejercer. [...] La sola y única condición que creo es necesaria para asegurar a la filosofia en el futuro próximo logros que sobrepasen todo lo alcanzado hasta hoy por los filósofos es la creación de una escuela de hombres con preparación científica y con intereses filosóficos, libres de tradiciones periclitadas y no extraviados por los métodos literarios de aquellos que copian todo de los antiguos excepto sus méritos (Russell, 1914, pp. 243-246) $)^{3}$.
Así, luego de haber adoptado el giro lingüístico, se suman, como influencias al pensamiento de Carnap, a los señalados neo-kantismo, positivismo crítico alemán, convencionalismo y fenomenología, la lógica de los Principia Mathematica de Whitehead y Russell y, vinculada con ésta, del análisis lógico del lenguaje de Frege y Wittgenstein. Si bien, "[m]ientras que los componentes lógicos y convencionalistas de su pensamiento ganan en importancia con el paso del tiempo, retroceden los aspectos neokantianos y fenomenológicos", aunque no desaparecen del todo, "sino que continúan actuando subterráneamente" (Mormann, 2000, 42).

El análisis lógico del lenguaje de Frege y Wittgenstein adquieren tal relevancia luego del giro lingüístico que en Carnap (1963a) aquéllos son mencionados, junto con Russell, como los de mayor influencia en su pensamiento. Sin embargo, ningún filósofo le causó mayor impacto a Carnap que Bertrand Russell. Luego de la lectura de Russell (1914) comentada más arriba, Carnap tuvo claro qué hacer en filosofía; el objetivo: analizar conceptos científicos y aclarar problemas filosóficos; el método: la aplicación de la nueva lógica de relaciones de los Principia Mathematica, o mejor aún "el uso de la lógica simbólica y de un sistema de lenguaje construido con reglas sintácticas y semánticas explícitas" (Carnap, 1963b, 936).

De hecho, toda la obra de Carnap puede ser dividida en dos: por un lado, discusión de las condiciones generales para la construcción o elaboración, y desarrollo -de acuerdo con ciertos fines perseguidos- y perfeccionamiento, de marcos lingüísticos ("semiótica pura", "teórica" o "formal"), en particular de sistemas formales ("semántica comprehensiva" o "lógica" en sentido amplio, que abarca tanto la lógica deductiva, como la intensional, la modal y la inductiva), y, por el otro, la aplicación de tales marcos lingüísticos (sistemas formales o "lógica") al campo no-lógico ("semiótica aplicada"), a fines de elucidación o análisis conceptual ("explication"4, en la terminología de Carnap 1950b)5.

Pero qué es lo que se debe hacer para llevar a cabo este tipo de análisis, y qué identifica Carnap con la labor filosófica, sufre diversas modificaciones a través de sus diferentes escritos. Así, se suele distinguir una etapa "sintáctica", que tiene su expresión más acabada en Carnap (1934a). De esta etapa es su concepción de la filosofía de la ciencia como "análisis lógico de los resultados lingüisticamente formulados de las acciones de los científicos", o, más brevemente, 
"análisis lógico del lenguaje de la ciencia" o "lógica de la ciencia", entendiendo por ésta sólo el estudio de la sintaxis lógica del lenguaje de la ciencia. Carnap explica la posición mantenida por él en esos años haciendo alusión al hecho histórico de que "el aspecto sintáctico del lenguaje ha sido el primero en ser investigado con medios exactos por Frege, Hilbert, los lógicos polacos y en mi libro" (Carnap, 1963a, 56). Alli, Carnap pretendió mostrar que las proposiciones respetables de la filosofía, como se formulan comúnmente, eran proposiciones sintácticas erróneamente expresadas en el modo material, ya que, a fin de expulsar el demonio de la metafisica (del sinsentido), era preciso definir expresiones como "significado", "verdad", "designación", en términos puramente sintácticos (esto es, como algo que hace referencia a las propiedades de oraciones de un sistema formal).

Sin embargo, ya desde hacía algunos años se estaba trabajando en una teoría sistemática que, con la ayuda de los instrumentos exactos de la lógica moderna, añadiéndose al análisis puramente formal del lenguaje, proporcionara un análisis de su función "significadora", esto es, fuera una teoría del significado y la interpretación. Esta teoría, llamada "semántica", si se desarrolla suficientemente, no sólo contiene una teoría de la relación de designación, e.e. de la relación entre las expresiones y sus significados, sino también una teoría de la verdad y una teoria de la deducción lógica ${ }^{6}$.

Una vez admitida la legitimidad de las investigaciones semánticas (Carnap 1935), Carnap se dedicó intensamente a ellas (Carnap, 1939, 1942, 1943, 1947/1956). En cuanto a la lógica de la ciencia, el análisis sintáctico-formal es complementado por el que toma en consideración la relación existente entre las expresiones lingüisticas y los objetos a los que éstas refieren, integrando, de este modo, tanto al análisis sintáctico como al semántico.

Como mencionamos previamente, la pragmática es -dentro de la teoría general de los signos y los lenguajes- la teoría de las relaciones entre los signos y aquéllos que los producen o reciben y entienden 8 .

Pero aun cuando el trabajo de Carnap se centra en los sistemas sintácticos y semánticos y sus relaciones ${ }^{9}$ y los elementos pragmáticos no fueran, o durante mucho tiempo no pudieran ser, ellos mismos objeto de análisis teórico, éstos siempre jugaron para Carnap (o les fue reconocido por él) un papel importante. Así, desde sus obras más tempranas (Carnap, 1922, 1928, 1934a), debido al peso del convencionalismo adoptado ${ }^{10}, 0$, más adelante (Carnap, 1950), con relación no tanto al análisis de los aspectos pragmáticos del lenguaje, sino al momento previo a la adopción/construcción de(I/un) lenguaje.

En ese sentido, podríamos decir que es conocida la distinción entre "cuestiones internas" y "cuestiones externas", presente en su clásico artículo (Carnap, 1950). Cuestiones internas son aquéllas que se plantean dentro de un lenguaje o marco lingüístico particular, mientras que externas son aquellas que se efectúan por fuera de todo marco lingüistico. Las cuestiones internas, a su vez, pueden dividirse en particulares y generales. Las cuestiones internas particulares pueden formularse si se establece un lenguaje con determinadas reglas (sintácticas y semánticas) y procedimientos de prueba. Si el marco lingüístico es empírico (del tipo del llamado "lenguaje-cosa" [thing-language] para objetos físicos), podemos preguntar, por ejemplo, "¿hay un cenicero sobre la mesa?" y la respuesta se determinará a través de métodos empíricos; si, en cambio, el marco no es empírico, como en el caso del lenguaje de números, a la cuestión interna particular "¿hay un número primo mayor que cien?" se responde mediante un análisis lógico, y las respuestas son analíticas. Las cuestiones internas generales (como "¿existen objetos físicos?" o "¿hay números?") se responden a partir de las respuestas afirmativas a las cuestiones internas particulares: si hay un cenicero sobre la mesa y el cenicero es un objeto físico, se sigue lógicamente que hay objetos físicos, y lo mismo ocurre con la respuesta a la cuestión sobre la existencia de los números. Sin embargo, cuando los filósofos tratan, por ejemplo, la cuestión de la existencia de los números o de la realidad del mundo de las cosas no están formulando una cuestión interna. Aunque estas cuestiones tradicionales se formulan de la misma manera que las cuestiones internas generales, no pertenecen a este tipo de cuestiones. Ellas están pensadas independientemente del aparato interno de un lenguaje $y$, por lo tanto, deben ser consideradas como externas. No pueden ser formuladas dentro del sistema porque se refieren al sistema como un todo y suelen plantearse antes de formular o aceptar un sistema particular. Carnap divide las cuestiones externas en prácticas (o pragmáticas) y teóricas. Las primeras se pueden formular con preguntas como "¿debe ser aceptado un lenguaje que contiene tales y tales formas lingüísticas?". Se trata de una cuestión cuya respuesta no es ni verdadera ni falsa, porque no es una 
aserción, sino que implica una evaluación de las ventajas y desventajas de aceptar un determinado marco lingüístico. Las respuestas a las cuestiones externas llevan a una decisión práctica (o pragmática). La decisión de aceptar un marco lingüístico puede estar influida por conocimientos teóricos, porque los propósitos con que se pretende usar un lenguaje determinarán los factores relevantes para tomar una decisión. De esta manera, la eficacia, fecundidad y simplicidad en el uso de un lenguaje pueden ser factores decisivos, y la evaluación de estas cualidades en un lenguaje es de naturaleza teórica. Las cuestiones internas particulares y generales y las cuestiones externas prácticas pueden plantearse legítimamente, mientras que las cuestiones externas teóricas, según Carnap, no han podido ser formuladas en términos del lenguaje científico, por lo cual sostiene que tanto ellas como sus respuestas carecen de sentido o contenido cognitivo; sólo adquieren significado si son tomadas como propuestas para la aceptación de una cierta forma de lenguaje.

Lo que es quizás menos conocido es que dicha distinción, aunque no de manera idéntica ni con todos los detalles, ya aparece en un trabajo muy anterior (Carnap, 1934b), en donde puede apreciarse la relevancia aun política y social de tal distinción y de la filosofía en general"

Allí Carnap nos habla del "ámbito de la decisión teórica" y de la "obligación de la toma de posición práctica", diciendo que:

[e]ntre ambas, sin embargo, hay una diferencia fundamental, sobre la cual debemos llegar a ser claros.

La ambigüedad del uso del lenguaje tiene aquí un efecto confundente, ya que alli también se habla de "cuestiones" [preguntas], donde en realidad no se plantean cuestiones [preguntas], esto es, demandas para decidir sobre lo verdadero y lo falso, sino situaciones, en las que se toma una decisión sobre el actuar (Carnap, 1934b, 259).

Sin embargo, lo que resulta especialmente interesante de este trabajo son las consecuencias prácticas que se señalan como siguiéndose de esta distinción, en donde éstas no se refieren a la aceptación o no de entidades abstractas en semántica, tales como propiedades, clases, relaciones, números, proposiciones, etc., sino a "ciertas consecuencias para las formas de la lucha que llevamos a cabo contra las supersticiones, la teología, la metafísica, la moral tradi- cional, la explotación capitalista de los trabajadores, etc." (Carnap, 1934b, 260). Asi se dice que ( $y$, debido a lo poco conocido de este artículo, me permito citarlo in extenso):

[e]n el caso de las supersticiones se trata de cuestiones teóricas; la aceptación de que los rezos y amuletos pueden proteger de los granizos y los accidentes de trenes pueden ser refutados científicamente. Si, por el contrario, alguien está a favor o en contra del socialismo, es asunto de la toma de posición práctica, no de la demostración teórica. Aquí sólo puede ser establecido teóricamente que esta y aquella organización tienen estas y aquellas consecuencias higiénicas, económicas y culturales. Esta es una preparación muy importante de nuestra toma de posición; pero no nos ahorramos por ello de esta toma de posición. Debemos decidir si queremos o no las consecuencias establecidas en la reflexión teórica (p.e. la superación de las crisis económicas y del desempleo); de ello depende entonces nuestro actuar, con base en la opinión teórica. La reflexión científica no determina el objetivo, sino siempre sólo el camino hacia el objetivo escogido. La metafísica es lírica en el ropaje de una teoría. Es una mera expresión de sentimientos, pero que mediante el vestuario lingüistico en enunciados aseverativos da la apariencia de que proporciona conocimiento. En la teología oficial y en las demás doctrinas religiosas se mezclan superstición y metafísica; se podría distinguir aquí entre teología "mitológica" y "metafísica". [...] Teóricamente sólo se deja comprobar que la metafisica filosófica y religiosa es, bajo ciertas circunstancias, un narcótico peligroso y que afecta la razón. Nosotros rechazamos ese narcótico. Si otros aman su disfrute, nosotros no lo podemos refutar teóricamente. Eso no significa de ningún modo, sin embargo, que nos debe ser indiferente cómo los hombres deciden en este punto. Podemos dar ilustración teórica sobre el origen y los efectos del narcótico. Además, podemos influir en ese punto por medio de la convocatoria, la educación y el ejemplo sobre la decisión práctica de los hombres. Sólo queremos ser claros sobre ello, que esa influencia reside fuera del ámbito teórico de la ciencia (Carnap, 1934b, 260).

Como ya ha sido señalado, una de las lamentables consecuencias del ascenso del nazismo y consiguiente emigración sobre el positivismo lógico y su desarrollo en los Estados Unidos fue la despolitización, siendo común a muchos de los intelectuales izquierdistas que arribaron a dicho país (p.e., Theodor Adorno) la "declinación del radicalismo", aun cuando es digno de observarse que muchos representantes 
del positivismo lógico, Carnap entre ellos, no rompieron como individuos con sus creencias anteriores ${ }^{12}$.

Pero el reconocimiento por parte de Carnap del importante papel de los elementos pragmáticos no sólo lo podemos encontrar en el ámbito de las decisiones prácticas -de las cuales la adopción o construcción de un lenguaje o marco lingüístico determinado constituye, como vimos, un caso particular-. También lo encontramos en relación con el análisis de la ciencia (en particular con el análisis de ésta en tanto práctica o actividad), pues, como nos dice, "las actividades de percepción, observación, comparación, registro, confirmación, etc. [son susceptibles de ser analizadas mediante la pragmática] en la medida en que estas actividades llevan o refieren a conocimiento formulado en lenguaje" (Carnap, 1942, p. 245). Incluso si consideramos la reinterpretación, subrayada por Stegmüller (1959, 1971, 1973a, 1973b), que da a la lógica inductiva en sus obras tardias sobre el tema (Carnap, 1959, 1962a, 1962b, 1971a, 1971b) desde el punto de vista de la teoría de la decisión racional, como proveyendo reglas o normas para la toma de decisiones racionales, y el hecho de que no sólo apoyó el desarrollo de una pragmática formal (p.e., del tipo que encontramos en las obras de Martin, 1959, y Montague, 1974), sino que él mismo realizó algunos aportes tanto al ámbito de la pragmática pura como de la aplicada (Carnap, 1955), también sería factible rastrear un cierto "giro pragmático" en Carnap (pero en donde, a diferencia del que también se puede detectar en Hempel, no está acompañado de "naturalismo"), aunque sin ánimos de magnificar su importancia respecto del conjunto de su obra, que sería predominantemente sintáctico-semántica.

\section{Balance y perspectivas futuras}

Hemos señalado la asociación que suele hacerse de los dos primeros períodos, épocas o fases de la filosofía de la ciencia del siglo XX a partir de su profesionalización, la "clásica" y la "histórica", con los denominados "giro lingüístico" y "giro pragmático", respectivamente. También hemos visto cómo dicha asociación podría ser útil como una primera aproximación, pero que, en realidad, habria que matizar dicho relato, quizás señalando que, en general, $y$, por lo tanto, no en todos los autores, se trata más bien de un problema de énfasis y/o de desarrollo o tratamiento sistemático. En particular, podriamos decir, por un lado -aun cuando esto no haya sido elaborado en este artículo-, que el "giro lingüístico", con su énfasis en el análisis de los aspectos sintácticos y semánticos del lenguaje de la ciencia, característico de la fase clásica, no dejó de estar presente en la fase histórica, si bien con cierto descuido respecto de las distinciones terminológicas o conceptuales precisas, e.e. de las elucidaciones conceptuales satisfactorias en relación con el grado de precisión alcanzado. Lo que sí vimos aquí fue cómo, por otro lado, en el propio Carnap encontramos muy tempranamente el reconocimiento del importante papel jugado, en distintos ámbitos, por elementos pragmáticos de diversa índole.

Si consideramos ahora a la filosofía contemporánea de la ciencia, en particular en la versión de la concepción estructuralista de las teorias, ya fue observado que nos encontramos tanto con intentos de desarrollar una elucidación de los conceptos (meta-) científicos (y de los marcos conceptuales científicos o teorías) lo más precisa posible, echando mano de todos los instrumentos formales disponibles, como con el reconocimiento de la presencia de elementos histórico-pragmáticos irreductibles (pero susceptibles de recibir un tratamiento riguroso, eventualmente, aunque no necesariamente, a través de la pragmática formal), es decir, con aspectos que intentan recuperar lo acentuado durante las fases "clásica" e "histórica", pero en un tratamiento que se supone mejorado, en una suerte de "Aufhebung" en sentido hegeliano o "síntesis superadora".

Por otra parte, hacia fines del siglo pasado, sin embargo, algunos autores pensaron que la filosofía de la ciencia debía ocuparse más de los factores que conducen a la formulación de teorias que de las teorías mismas, y, en general, más de la práctica científica, buscando establecer así las bases conceptuales de una filosofía de la práctica científica. Este enfoque, que pone en el centro del análisis del conocimiento el concepto de práctica, se asocia, en las ciencias sociales, con las propuestas de, entre otros, Marx, Durkheim, Weber, Giddens y, particularmente, Bourdieu, y ha sido impulsado por historiadores (Franklin, 1986; Galison, 1987), sociólogos (Latour, 1987; Pickering, 1995; Schatzki, Knorr-Cetina \& von Savigny, 2001) y filósofos de la ciencia (Ackermann, 1985; Hacking, 1983; Buchwald, 1995; Echeverria, 2002; Fuller, 1989, 1991; Rouse, 1987, 1996, 2002; Schatzki, Knorr-Cetina \& von Savigny, 2001). Es así que -y en la medida en que la actividad científica 
involucre una serie de prácticas convencionales realizadas de acuerdo con ciertas reglas, normas o convenciones (aun cuando no haya un conocimiento explícito o consciente de las reglas involucradas, sino sólo tácito, implícito o inconsciente) y en que, si bien no para practicar la ciencia correctamente, aunque sí para saber en qué consiste llevarla a cabo, es necesario conocer las reglas que gobiernan dicha práctica- para este enfoque la filosofía de la ciencia tiene por finalidad hacer explícitas las reglas que rigen las diversas prácticas, de esa actividad que es hacer ciencia, haciéndola de este modo comprensible.

Estos desarrollos pueden verse como parcialmente vinculados con la adopción por parte de la filosofía historicista de la ciencia de aquello que se ha denominado "giro pragmático", aun cuando estos teóricos frecuentemente han sido ambivalentes acerca de la importancia del lenguaje para las prácticas, y viceversa: por un lado, las prácticas desarticuladas o incluso inarticulables son frecuentemente contrastadas con afirmaciones o reglas explícitas; por otro lado, el dominio de la práctica generalmente es extendido de modo de incorporar prácticas conceptuales 0 lingüisticas, quizás incluso como caso paradigmático de las prácticas (Rouse, 2001).

Además, una filosofía de la ciencia centrada en el análisis de las prácticas científicas no tiene porqué ser incompatible con la filosofía de la ciencia centrada en el análisis del conocimiento científico, en especial en el expresado en las teorias científicas. Nuevamente, si pensamos en la metateoría estructuralista, en ella se reconoce que, para poder llevar a cabo sus actividades, así como también para plasmar sus resultados, los científicos se sirven, entre otras cosas, del lenguaje. Sin embargo, está claro que, al no ser lingüisticas o verbales todas las acciones o conductas en general, ni de los científicos en particular, y, por lo tanto, susceptibles de análisis semiótico vía pragmática, aun cuando pudieran realizarse de acuerdo con convenciones, reglas o normas explícitas, o implícitas, pero explicitables, lingüisticamente, la propuesta de Carnap, a la luz de la filosofía contemporánea de la ciencia, que asume como tarea legítima el análisis de la práctica científica en su acepción más amplia, que incluye acciones de diversa índole y no se encuentra restringida a las acciones o conductas lingüisticas (o verbales), o sea, al uso que los científicos hacen del lenguaje de la ciencia, nos parece no del todo satisfactoria, pero por incompleta, teniendo que complementarla, dependiendo del desarrollo particular escogido, con una pragmática (de la ciencia) ampliada, una teoría de la acción (científica) -ya sea racional, individual, colectiva o social- o una praxiología (de la ciencia) ${ }^{13}$.

\section{AGRADECIMIENTOS}

Este trabajo ha sido realizado con la ayuda de los proyectos de investigación PICT Redes 2002 N. ${ }^{\circ} 00219$, PICT2003 N. 14261 y PICT Redes 2006 N. 2007 de la Agencia Nacional de Promoción Científica y Tecnológica. Quisiera agradecer a Andrés Rivadulla, Carlos Castrodeza, César Lorenzano, Luis Vega y Ricardo Gómez sus comentarios a versiones previas. Asimismo, quisiera agradecer a Friedrich Stadler, Director Científico del Institut Wiener Kreis, por haberme facilitado una copia del texto de Carnap "Theoretische Fragen u. praktische Entscheidungen" (Carnap, 1934b).

\section{NOTAS}

1 Que se encontraba en consonancia y relacionado con individuos y grupos afines de Viena, Praga, Alemania, PoIonia, los países escandinavos, Italia, Francia, Inglaterra, Estados Unidos y aun China.

2 Mención aparte -por su relevancia y por no pertenecer al Círculo de Viena,

Recibido: 14 de junio de 2009

Aceptado: 3 de diciembre de 2009 aunque estando en contacto con él, además de con otros miembros de la escuela polaca de filosofía de la ciencia- merece el investigador biomédico y filósofo e historiador de la ciencia, el judío polaco Luwik Fleck, cuyo Fleck (1935), anticipa muchas de las ideas de Kuhn, según este último reconoce en el "Prefacio" a su obra más importante y conocida (Kuhn, 1962, 11-12).

3 En el prefacio, Russell le atribuye a Whitehead, entre otras, "toda la concepción del mundo de la física como una construcción más bien que 
como una inferencia" (Russell, 1914, 8), es decir, en términos de Richardson, toda la idea detrás de la "máxima suprema del filosofar científico" (Richardson, 1998, n. 12, 14), cuya formulación más conocida ("La máxima suprema en el filosofar científico es ésta: siempre que sea posible, las entidades inferidas deben ser sustituidas por construcciones lógicas", Russell, 1914b, reimpresa en Russell 1917) constituye el epígrafe de Carnap (1928). Asimismo, habria que mencionar que, al comienzo mismo de dicho prefacio, Russell afirma que el "primer ejemplo completo [del método lógico-analítico en la filosofía] se encuentra en los escritos de Frege" (Russell, 1914, 7).

4 La elucidación o análisis conceptual ("explication") consiste en la transformación de un concepto dado más o menos inexacto o impreciso (el explicandum) en otro nuevo (el explicatum) exacto o preciso, o al menos más exacto o preciso que el anterior, 0 , más bien, en el reemplazo o sustitución del primero por el segundo, en donde el explicatum debe satisfacer los requisitos de semejanza con el explicandum, exactitud, fertilidad y simplicidad, y de la cual no decimos que sea verdadera o falsa o correcta o errónea, sino satisfactoria (o adecuada) o no o más satisfactoria (o adecuada) que otra (Carnap, 1950b).

5 Apoyo a esta lectura de la obra de Carnap, la encontramos en las propias reflexiones metafilosóficas que él efectúa. En Carnap (1963a), escribe: "una de las tareas más importantes de los filósofos es investigar las diversas formas de lenguaje posibles y descubrir sus propiedades características" (87), hace hincapié en el primero de los aspectos, mientras que en el prefacio a la segunda edición de su primera gran obra (Carnap, 1928), fechado en marzo de 1961, al afirmar que "[la] clarificación de conceptos, hoy en día llamada frecuentemente 'elucidación', todavía me parece una de las tareas más importantes de la filosofia" (Carnap, 1928/1961, v), enfatiza el segundo. Ambos aspectos también los encontramos en el siguiente pasaje: "En períodos tempranos, a menudo hice intentos de dar una elucidación del término 'filosofía.' El dominio de aquellos problemas que propuse llamar 'filosóficos' llegaron a ser poco a poco más comprehensivos, como señala Morris. Sin embargo, ninguna de mis elucidaciones me pareció en realidad completamente satisfactoria, incluso cuando las propuse; y tampoco me gustaron más las elucidaciones propuestas por otros. Por último, abandoné la búsqueda. Acuerdo con Morris en que no es sabio intentar una elucidación tal, debido a que cada una de ellas es más o menos artificial. Parece mejor dejar el término 'filosofia' sin límites precisos y sólo proponer la inclusión o la exclusión de ciertos problemas.

En particular, muchos problemas concernientes a los marcos conceptuales me parecen pertenecer a los problemas más importantes de la filosofía. Pienso aquí tanto en las investigaciones teóricas como en las deliberaciones prácticas y decisiones con respecto a una aceptación o un cambio de los marcos, especialmente de los marcos más generales que contienen los conceptos categoriales que son fundamentales para la representación de todo conocimiento" (Carnap, 1963a, 862).

6 Los pasos en esta dirección los habian venido dando los lógicos de la escuela de Varsovia, en especial Lesniewski y Kotarbiński, desde 1919. Pero no fue sino hasta la obra de otro lógico polaco, Alfred Tarski, que se echaron las bases para una construcción sistemática de una teoria tal. Su fundamental trabajo sobre el concepto de verdad (Tarski, 1935), publicado originariamente en polaco en 1933, así como el desarrollo completo de las investigaciones semánticas en Polonia, permanecieron desconocidas para el mundo exterior hasta 1936, salvo para unos pocos elegidos, entre los que se encontraba Carnap, que tuvieron noticias de esto con algún tiempo de anticipación.

7 Hintikka (1975, 1992), por su parte, afirma que no es sostenible una distinción estricta entre período "sintáctico" (hasta 1934) y "semántico" (a partir de 1935), ya que en la obra más significativa del período "sintáctico" (Carnap, 1934a), hay mucho de lo que ahora se considera semántico (de hecho, la mayoría de los resultados de Logische Syntax permanecen como válidos en su obra semántica posterior), por lo cual el llamado período "semántico" debería ser considerado como una generalización y ampliación no especialmente dramática de su punto de vista sintáctico.

8 En el desarrollo de una teoría tal son pioneros los trabajos de Peirce (1931), Ogden y Richards (1923) y Morris (1938). La importancia de dicha teoría fue reconocida por Carnap a través de su relación con el pragmatismo, relación que se inició en 1934 al encontrarse con dos de sus jóvenes seguidores, Nagel y Morris, en el Congreso Internacional de Filosofía realizado en Praga, y que se vio estrechada al trasladarse en 1936 a los Estados Unidos.

9 Desarrollando las sintaxis y semántica puras y generales: puras en tanto 
que analíticas y sin contenido empírico, comprendiendo la construcción y el análisis de los sistemas sintácticos y semánticos, y en el que se establecen las reglas relativas a dichos sistemas, formulando definiciones de cierta clase de conceptos y estipulando sus consecuencias, por oposición a un análisis descriptivo, que, al consistir en una investigación empírica de las características semánticas de los lenguajes históricamente dados, es una ciencia empírica; y generales en tanto que comprende todo lenguaje históricamente dado o todo sistema sintáctico y semántico, en lugar de ser especial y concentrarse así en un lenguaje o sistema semántico particular.

10 Convencionalismo que, frente al lenguaje formal privilegiado de los Principia Mathematica de Russell y Whitehead (1910-1913) adoptado en sus trabajos previos, recién se extendería al campo de la lógica bajo la forma del conocido "principio de tolerancia" (Carnap, 1934a) y que en su forma más general podría ser llamado "principio de la convencionalidad de las formas de lenguaje" (Carnap, 1963a, 55).

11 Para los aspectos políticos y sociales del Círculo de Viena, se puede ver, p.e., Wartofsky (1982).

12 Para un análisis del contexto en el cual tiene lugar el cambio aludido, ver Reisch (2005).

13 Un ejemplo de análisis de estructuras no lingüisticas (ni habitualmente formuladas lingüisticamente), pero susceptibles de ser identificadas a través de (sí el lenguaje, pero, fundamentalmente de) la práctica científica, tornándola comprensible, es el realizado en Lorenzano (2006) de la teoria genética y de la práctica que los genetistas que disponen de dicha teoría llevan a cabo, mediada por la (nunca explícitamente formulada) ley fundamental de esa teoría.

\section{BIBLIOGRAFÍA}

Ackermann, Robert John (1985): Data, Instruments, and Theory, Princeton: Princeton University Press.

Bergmann, Gustav (1964): Logic and Reality, Madison: The University of Wisconsin Press.

Buchwald, Jed Z. (ed.) (1995): Scientific Practice: Theories and Stories of Doing Physics, Chicago: University of Chicago Press.

Carnap, Rudolf (1922): Der Raum. Ein Beitrag zur Wissenschaftslehre, Berlin: Verlag von Reuther \& Reichard.

Carnap, Rudolf (1928): Der logische Aufbau der Welt, Berlin: Welkreis-Verlag, 2. ${ }^{a}$ ed. con Scheinprobleme in der Philosophie, "Vorwort zum 2. Auflage" y "Literaturverzeichnis 1961", Hamburg: Felix Meiner Verlag, 1961.

Carnap, Rudolf (1934a): Logische Syntax der Sprache, Vienna: Springer.

Carnap, Rudolf (1934b): "Theoretische Fragen u. praktische Entscheidungen", Natur und Geist, 9: 257-260.

Carnap, R. (1935): "Warheit und Bewährung", Actes du Congrès International de Philosophie Scientifique, Sorbonne, Paris, vol. IV Induction et Probabilité, Paris: Hermann \& Cie., Éditeurs, 1936, pp. 18-23.

Carnap, Rudolf (1939): Foundations of Logic and Mathematics. Encyclopedia of Unified Science, vol. 1, no. 3, Chicago: University of Chicago Press.

Carnap, Rudolf (1942): Introduction to Semantics, Cambridge, Mass.: Harvard University Press.

Carnap, Rudolf (1943): Formalization of Logic, Cambridge, Mass.: Cambridge University Press.
Carnap, Rudolf (1947): Meaning and Necessity. A Study in Semantics and Modal Logic, Chicago: University of

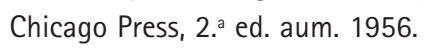

Carnap, Rudolf (1950a): "Empiricism, Semantics and Ontology", Revue Internationale de Philosophie, 4: 20-40.

Carnap, Rudolf (1950b): "On Explication", Logical Foundations of Probability, Chicago: University of Chicago Press, Cap. 1, pp. 1-18.

Carnap, Rudolf (1955): "On Some Concepts of Pragmatics", Philosophical Studies, 6: 89-91.

Carnap, Rudolf (1959): Induktive Logik und Wahrscheinlichkeit, bearbeitet von W. Stegmüller, Wien: Springer.

Carnap, Rudolf (1962a): "The Aim of Inductive Logic", en Nagel, Ernest, Patrick Suppes y Alfred Tarski (eds.), Logic, Methodology and Philosophy of Science, Stanford: Stanford University Press, 303-318.

Carnap, Rudolf (1962b): "Preface to the Second Edition", en Carnap, Rudolf, Logical Foundations of Probability, Chicago: University of Chicago Press, second edition, pp. xiii-xxii.

Carnap, Rudolf (1963a): "Intellectual Autobiography", en Schilpp, Paul Arthur (ed.), The Philosophy of Rudolf Carnap, La Salle, III.: Open Court, 1963, pp. 1-84.

Carnap, Rudolf (1963b), "Replies and Systematic Expositions", en Schilpp, Paul Arthur (ed.), The Philosophy of Rudolf Carnap, La Salle, III.: Open Court, 1963, pp. 859-1013.

Carnap, Rudolf (1971a), "Inductive Logic and Rational Decisions", en Carnap, Rudolf y Richard C. Jeffrey (eds.), Studies in Inductive Logic and Probability, Vol. I, Berkekey, California: University of California Press, 5-31.

Carnap, Rudolf (1971b): "A Basic System of Inductive Logic, Part I", en Carnap, Rudolf y Richard C. Jeffrey (eds.), Stu- 
dies in Inductive Logic and Probability, Vol. I, Berkekey, California: University of California Press, 33-165.

Carnap, R., O. Neurath y H. Hahn (1929): Wissenschaftliche Weltauffassung. Der Wiener Kreis, Wien: Artur Wolf Verlag. Traducción castellana: "La concepción científica del mundo: el Círculo de Viena", Redes. Revista de Estudios sobre la Ciencia y la Tecnología 18 (2002): 103-149.

Coffa, J. Alberto (1991): The Semantic Tradition from Kant to Carnap: To the Vienna Station, edited by Linda Wessels. Cambridge: Cambridge University Press.

Dummett, Michael (1993): Origins of Analytical Philosophy, Cambridge, Massachusetts: Oxford University Press.

Echeverria, Javier (2002): Ciencia y valores, Barcelona: Destino.

Fleck, Ludwik (1935): Entstehung und Entwicklung einer wissenschaftlichen Tatsache. Einführung in die Lehre vom Denskstil und Denkkollektiv, Basel: Benno Schwabe \& Co.

Franklin, Allan (1986): The Neglect of Experiment, Cambridge: Cambridge University Press, 1986.

Fuller, Steve (1989): Philosophy of Science and its Discontents, Boulder: Westview.

Fuller, Steve (1993): Philosophy, Rhetoric, and the End of Knowledge, Madison: University of Wisconsin Press.

Galison, Peter (1987): How Experiments End, Chicago: Chicago University Press.

Hacking, lan (1983): Representing and Intervening, Cambridge: Cambridge University Press.

Hempel, Carl Gustav (1979a): "Scientific Rationality: Analytic vs Pragmatic Perspectives", en Geraets, Theodore (ed.), Rationality Today, Ottawa: University of Ottawa Press, pp. 46-58.
Hempel, Carl Gustav (1979b): "Scientific Rationality: Normative vs Descriptive Construals", en Berghel, Hal, Adolf Hübner y Eckehart Köhler (eds.), Wittgenstein, the Vienna Circle, and Critical Rationalism, Vienna: HoelderPichler-Tempsky, pp. 291-301.

Hempel, Carl Gustav (1981): "Turns in the Evolution of the Problem of Induction", Synthese, 46: 389-404.

Hempel, Carl Gustav (1982): "Schlick und Neurath: Fundierung vs. Kohärenz in der wissenschaftlichen Erkenntnis", Grazer philosophische Studien 16/17: 1-18.

Hempel, Carl Gustav (1983): "Valuation and Objectivity in Science", en Robert S. Cohen y Larry Laudan (eds.), Physics, Philosophy and Psychoanalysis, Dordrecht: Reidel, pp. 73-100.

Hempel, Carl Gustav (1993): "Thomas Kuhn, Colleage and Friend", en Horwich, Paul (ed.), World Changes. Thomas Kuhn and the Nature of Science, Cambridge, Massachusetts: The MIT Press, pp. 7-8.

Hintikka, Jaakko (1975): "Carnap's Heritage in Logical Semantics", en Hintikka, Jaakko (ed.), Rudolf Carnap, Logical Empiricist, Dordrecht: Reidel, pp. 217242.

Hintikka, Jaakko (1992), "Carnap's Work in the Foundations of Logic and Mathematics in a Historical Perspective", Synthese, 93: 167-189.

Kambartel, Friedrich (1968): Erfahrung und Struktur. Bausteine zu einer Kritik des Empirismus und Formalismus, Frankfurt am Main: Suhrkamp.

Kuhn, Thomas S. (1962): The Structure of Scientific Revolutions, Chicago University Press, Chicago, 2. ${ }^{\text {a }}$ edición 1970.

Latour, Bruno (1987): Science In Action: How to Follow Scientists and Engineers Through Society, Cambridge, Mass.: Harvard University Press.
Lorenzano, Pablo (2006): "Fundamental Laws and Laws of Biology", en Ernst, Gerhard y Karl-Georg Niebergall (eds.), Philosophie der Wissenschaft - Wissenschaft der Philosophie. Festschrift für C. Ulises Moulines zum 60. Geburstag, Paderborn: Mentis-Verlag, pp. 129-155.

Martin, Richard M. (1959): Toward a Systematic Pragmatics, Amsterdam: NorthHolland.

Montague, Richard (1974): Formal Philosophy. Selected Papers of Richard Montague, New Haven/London: Yale University Press.

Mormann, Thomas (2000): Rudolf Carnap, München: Beck.

Morris, Charles William (1938): "Foundations of the Theory of Signs", en Neurath, Otto (ed.), International Encyclopedia of Unified Science, vol. 1, no. 2, Chicago: University of Chicago Press.

Moulines, Carlos Ulises (2002): “¿Dónde se agazapa la pragmática en la representación estructural de las teorías?", en Díez, José Antonio y Pablo Lorenzano (eds.), Desarrollos actuales de la metateoría estructuralista: problemas y discusiones, Quilmes: Universidad Rovira i Virgili/Universidad Autónoma de Zacatecas/Universidad Nacional de Quilmes, 2002, pp. 99-115.

Neurath, Otto (1979): Wissenschaftliche Weltauffassung, Sozialismus und Logische Empirismus, ed. por Rainer Hegselmann, Frankfurt am Main: Suhrkamp.

Neurath, Otto (1981): Gesammelte philosophische und methodologische Schriften, ed. por Rudolf Haller y Heiner Rutte, 2 tomos, Wien: HoelderPichler-Tempsky.

Neurath, Otto (1991): Gesammelte bildpädagogische Schriften, ed. por Rudolf Haller y R. Kinross, Wien: HoelderPichler-Tempsky. 
Neurath, Otto (1994): Otto Neurath oder Die Einheit von Wissenschaft und Gesellschaft, ed. por Paul Neurath und Elisabeth Nemeth, Wien-Köln-Weimar: Böhlau.

Neurath, Otto (1998): Gesammelte ökonomische und sozialpolitische Schriften, ed. por R. Haller y Ulf Höfer, 2 tomos, Wien: Hoelder-Pichler-Tempsky.

Ogden, Charles Kay e Ivor Armstrong Richards (1923): The Meaning of "Meaning": A Study of the Influence of Language Upon Thought and of the Science of Symbolism, London: Routledge \& Kegan Paul.

Peirce, Charles Saunders (1931): Collected Papers, vol. II, Cambridge, Mass.: Harvard University Press.

Pickering, Andrew (1995), The Mangle of Practice, Chicago: University of Chicago Press.

Quine, Willard Van Orman (1951): "Two Dogmas of Empiricism", Philosophical Review, 60: 20-43.

Reisch, George A. (2005): How The Cold War Transformed Philosophy of Science: To the Icy Slopes of Logic, Cambridge: Cambridge University Press.

Rouse, Joseph (1987): Knowledge and Power: Toward a Political Philosophy of Science, Ithaca and London: Cornell University Press.

Rouse, Joseph (1996): Engaging Science: Science Studies after Realism, Rationality, and Social Constructivism, Cornell: Cornell University Press.

Rouse, Joseph (2002): How Scientific Practices Matter: Reclaiming Philosophical Naturalism, Chicago: Chicago University Press.

Russell, Bertrand (1914): Our Knowledge of the External World, Chicago: Open Court.
Russell, Bertrand y Alfred North Whitehead (1910-1913): Principia Mathematica, 3 vols., Cambridge: Cambridge University Press.

Schatzki, Theodore R., Karin Knorr-Cetina y Eike von Savigny (eds.) (2001): The Practice Turn in Contemporary Theory, London: Routledge.

Stegmüller, Wolfgang (1959): "Carnaps Auffassung der induktiven Logik", en Carnap, Rudolf, Induktive Logik und Wahrscheinlichkeit, bearbeitet von Wolfgang Stegmüller, Wien: Springer.

Stegmüller, Wolfgang (1970): Theorie und Erfahrung, Berlin: Springer.

Stegmüller, Wolfgang (1971): "Das Problem der Induktion: Humes Herausforderung und moderne Antworten", en Lenk, Hans (ed.), Neue Aspekte der Wissenschaftstheorie, Braunschweig.

Stegmüller, Wolfgang (1973a): Probleme und Resultate der Wissenschaftstheorie und der Analytischen Philosophie, Band IV: Personelle und Statistische Wahrscheinlichkeit, Erster Halbband: Personelle Wahrscheinlichkeit und Rationale Entscheidung, Berlin-Heidelberg-New York: Springer.

Stegmüller, Wolfgang (1973b): "Carnap's Normative Theory of Inductive Probability", en Suppes, Patrick Henkin, Leon, Joja, Athanese y Grigore C. Moisil (eds.), Logic, Methodology and Philosophy of Science IV, Amsterdam: North-Holland, 501-513.

Stegmüller, Wolfgang (1983a): Probleme und Resultate der Wissenschaftstheorie und Analytischen Philosophie, Band 1: Erklärung-Begründung-Kausalität, zweite, verbesserte und erwei- terte Auflage, Berlin-Heidelberg-New York: Springer-Verlag.

Stegmüller, Wolfgang (1983b): "Vom dritten bis sechsten (siebten?) Dogma des Empirismus", Information Philosophie, 4: 4-24.

Tarski, Alfred (1935): "Der Wahrheitsbegriff in den formalisierten Sprachen", Studia Philosophica, I: 261-405.

Wartofsky, M. W. (1982): "Positivism and Politics. The Vienna Circle as a Social Movement", en Haller, R. (ed.), SchlickNeurath. Ein Symposium, Amsterdam: Rodopi (Grazer philosophische Studien 16/17), pp. 79-101.

Wolters, Gereon (2003): "Carl Gustav Hempel: Pragmatic Empiricist", en Parrini, Paolo, Wesley Salmon y Merrilee Salmon (eds.), 2003, Logical Empiricism. Historical and Contemporary Perspectives, Pittsburgh: University of Pittsburgh Press, pp. 109-122.

Zilsel, Edgar (1918): Die Geniereligion. Ein kritischer Versuch über das moderne Persönlichkeitsideal mit einer historischen Begründung, Wien: Braumüller.

Zilsel, Edgar (1926): Die Entstehung des Geniebegriffes, Tübingen: J.C.B. Mohr.

Zilsel, Edgar (1976): Die sozialen Ursprünge der neuzeitlichen Wissenschaft, ed. y trad. por Wolfgang Krohn, Frankfurt am Main: Suhrkamp.

Zilsel, Edgar (1992): Wissenschaft und Weltanschauung: Aufsätze 19291933, ed. por Gerald Mozetič?, WienKöln-Weimar: Böhlau.

Zilsel, Edgar (2000): The Social Origins of Modern Science, ed. por Diderick Raven, Wolfgang Krohn y Robert S. Cohen, Dordrecht-Boston-London: Kluwer Academic Publishers. 